\title{
PROVIDING KNOWLEDGE SERVICES IN A CHALLENGING ENVIRONMENT: EXPERIENCES OF PRACTICAL ANSWERS SRI LANKA
}

\author{
Chandrika Kularathna \\ Practical Answers Sri Lanka, Practical Action Sri Lanka \\ Chandrika.ranasinghe@practicalaction.org.lk
}

\begin{abstract}
Financial sustainability is a key challenge which any donor funded knowledge sharing service as "Practical Answers Sri Lanka" has to face inevitably. Most institutions running with donor funds tend to discontinue the affiliated knowledge services or reduce the funding allocations considering them as cost entities. Hence it is very important to investigate innovative strategies, ownership models and unique management systems to ensure financial sustainability, while continuing to maximize the expected social impact through sharing knowledge.Financial sustainability is a challenge which Practical Answers Sri Lanka is striving to address in the long term. Practical Answers Sri Lanka adopted a new strategy to expand its vital services while moving the organizations towards self-sufficiency. In this paper the author presents the experiences of Practical Answers Sri Lanka in the process of transformation towards a sustainable knowledge sharing service.
\end{abstract}

Keywords: Financial Sustainability, Social Enterprise, Knowledge Sharing Services, Challenging Environment

\section{Introduction}

\subsection{Practical Action Group}

Practical Action is an UK based international non-governmental organization (INGO) that uses technology to challenge poverty in developing countries across the globe, founded in 1966 by the renowned economist Dr. E F Schumacher. Practical Action uses cost-effective, appropriate, small-scale development solutions to help people to help themselves. Practical Action works directly in more than 40 developing countries across the world. It has country and regional offices in Kenya, Zimbabwe, Peru, Sudan, Nepal, Sri Lanka, and Bangladesh, and its consultancy work extends across Africa, Asia and Latin America (www.practicalaction.org). 


\subsection{Practical Answers as a global service}

Practical Answers is the Technical Information Service of Practical Action. It provides information on appropriate, small-scale technologies that improve the lives of people living in poverty. Also it enables accessing the wealth of technical knowledge acquired within Practical Action over 47 years as well as the knowledge of its partners and other development organizations.

Practical Answers is a global service and it works across each country office in Bangladesh, Nepal, Sri Lanka, Kenya, Sudan, Zimbabwe, Peru, and United Kingdom. Practical answers' focus is providing information on technologies to communities, development workers, academics and to the public in general. Partnerships are fundamental to the work of Practical Answers. Hence Practical Answers work in collaboration with other organizations and specialists to collect and bring the technical knowledge and experience together through partnerships.

Practical Answers working model (figure 1) reflects two way flows of knowledge and demand for knowledge (Practical Action, 2012). Practical Action has vast knowledge base which consists of knowledge that originates from Practical Action work and the work of the partners and networks. Practical Answers adds value to such knowledge and information by formatting and adding accompanying information and disseminating this knowledge through its technical enquiry service, online platforms, and resource centers and through partners and networks. This shared technical information is expected to be adopted in providing solutions to technology related problems and changing the existing practices while ultimately helping to improve the wellbeing of the target communities.

\subsection{Practical Answers Sri Lanka (PASL) and its evolution}

Practical Action Sri Lanka represents the Practical Actions presence in Sri Lanka and began almost 25 years ago with a vision of a sustainable world free of poverty and injustice in which technology used for the benefit of all (Practical Action, 2012). Since its inception, Practical Action has tested and introduced several appropriate and intermediate technology options in its core subject areas such as renewable energy, agriculture and food processing, disaster management, housing, water and sanitation, small and medium enterprises and waste management. It worked with marginalized communities in all around Sri Lanka and some parts of India and Pakistan. 


\section{Practical Answers Working Model}

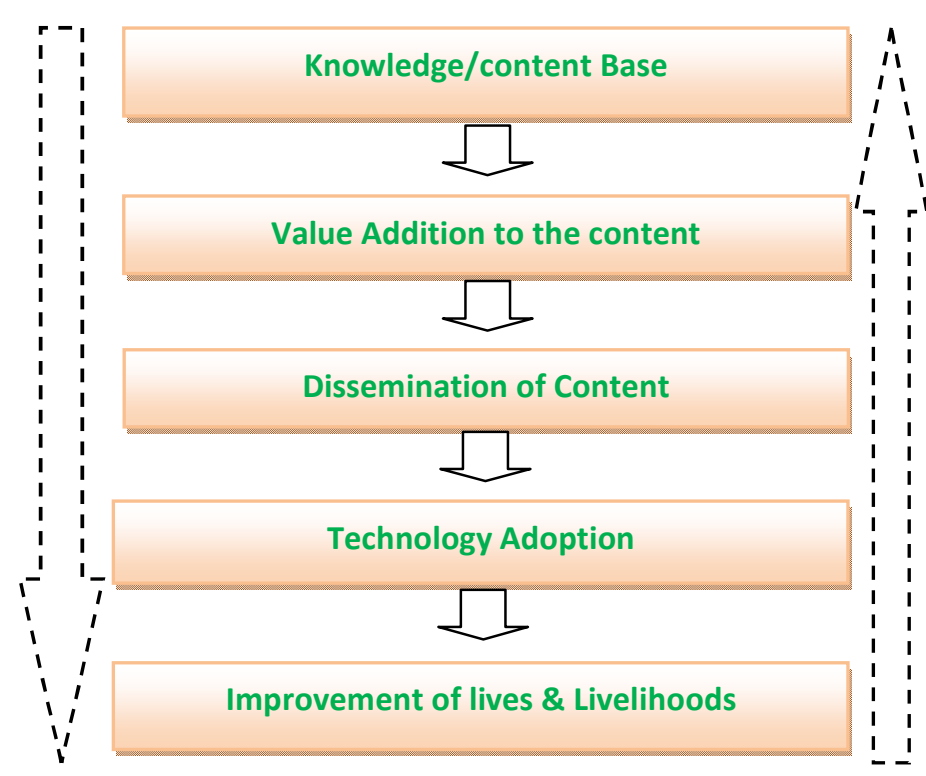

Figure 1: The working model of Practical Answers

Practical Action has a proud history of sharing knowledge about appropriate technologies which contributes to the development of the country. The technical enquiry service of Practical Action Sri Lanka was established along with establishment of a country office in Sri Lanka. The primary objective of this service was to provide the technical information, learning and experience with the people who need that information. Parallel to the technical inquiry service, the resource centre of Practical Action Sri Lanka was established to provide wider access to its wealth of knowledge and to support its project and programmes by providing the required information. These knowledge services of Practical Action Sri Lanka have widely grown to cover the whole island and some parts of India and Pakistan. In 2005, the knowledge portal of Practical Action Sri Lanka was developed with the primary objective of fulfilling the information needs of the post Tsunami rebuilding process and ultimately the portal become the online knowledge bank of Practical Action Sri Lanka.

Recognizing the importance of organizing all its knowledge services to deliver a more effective information service, Practical Action group established Practical Answers as the official knowledge service of Practical Action. The Practical Answers service was established in every country office of Practical Action and that is the formal start of practical answers Sri Lanka. At the beginning, Practical Answers Sri Lanka had four main knowledge provision components; technical enquiry service, resource centre, web portal and production unit which develop knowledge materials based on its' knowledge and experiences.

\subsection{Challenging Environment and the Need to Change}

Long term sustainability is a key concern that any non-profit organization continuously has to address. Sustainability is a challenge which Practical Answers also strive to address. 
Continuous fundraising is one strategy adopted by Practical Answers to meet this challenge of sustainability for the growth of Practical Answers. Trialling income generating initiatives was another strategy which Practical Answers wants to apply to face this challenge. Since 2011 PASL was trialling this by converting technical enquiries into income generating opportunities. PASL realized that there is a considerable potential to generate more income by expanding its knowledge services in to a new arena that is; providing training, capacity building and providing technological services although it is beyond the mandate of Practical Answers. The importance of the expansion of services of PASL to generate more income for self-sustainability was stressed by the announcement of the closure of Practical Action Sri Lanka by March 2014. To assure the sustainability of PASL in this highly uncertain and challenging environment, strategic decision to transform PASL into a social enterprise was taken. This decision was taken as a collective decision of the PASL team, PASL and Practical Action group management. The decision gave PASL an opportunity to expand its vital services while moving towards self-sufficiency,

\section{Strategies and Tools for Transformation}

To become a sustainable and effective knowledge service in a highly uncertain internal environment was a challenge to PASL and it had to develop a clear understanding how the existing resources and processes can be restructured to achieve sustainability. Practical Answers Sri Lanka also had to adopt a prospective business model, restructure its operations and services, develop a new marketing strategy, build new partnerships and closely monitor its impact. PASL has now started with this process and is closely monitoring and revising these strategies for a smooth transition toward a sustainable social enterprise by the end of year 2017.

\subsection{Hybrid business model, operations and organizational structure}

Transformation of a fully funded knowledge sharing service into a social enterprise was a formidable challenge. Practical Answers Sri Lanka has to adopt a new business model which delivers the expected social impact of the knowledge sharing and financial targets to achieve sustainability. Practical Answers Sri Lanka identified its comparative advantages, grabbed the opportunities in the internal and external environment, conducted a feasibility analysis and developed a new business model (figure 2) through expanding the existing services into viable businesses and introducing new businesses. PASL's business model is built on two main operating/service lines, where PASL generates revenues by providing services (Consultancy arm) in order to subsidize PASL's knowledge sharing services (technical enquiry desk, knowledge hub) free of charge. 


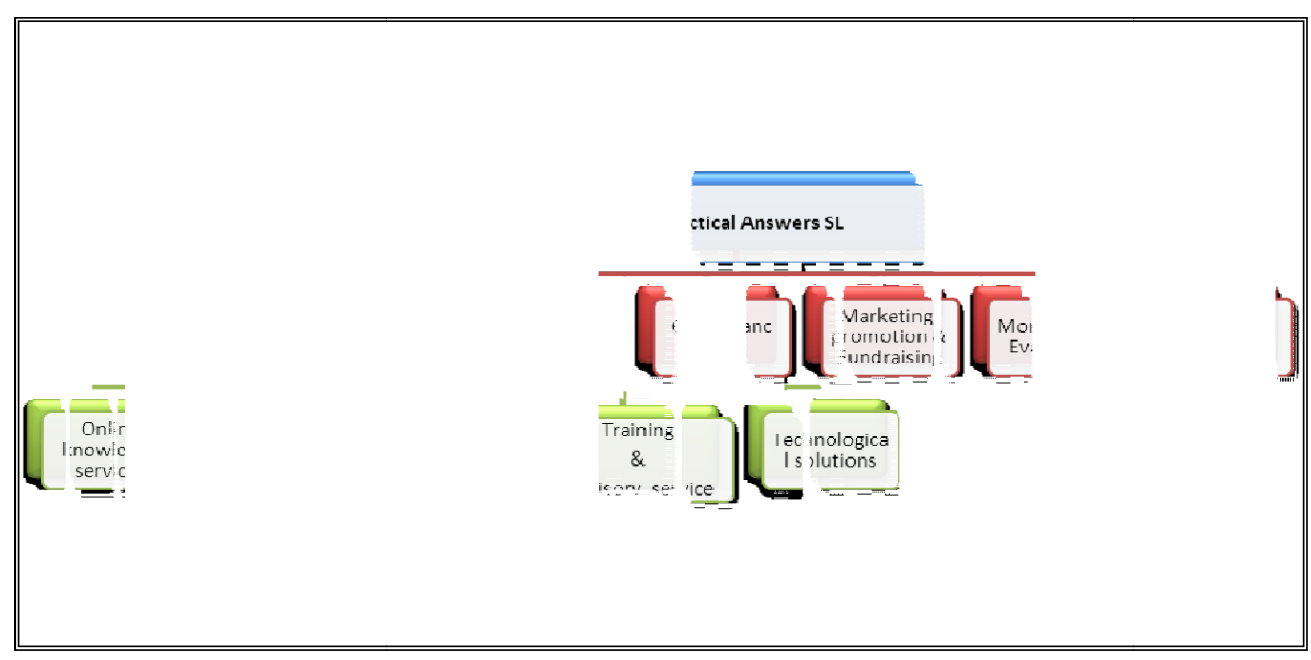

Figure 2: The functional units of PASL

The Technical Enquiry desk is the public interface of PASL. It handles the enquiries received from different audiences. It receives enquiries, directly answers selected enquiries, processes and direct some enquiries to relevant divisions such as its' consultancy arm and knowledge hub. It maintains enquiry databases and continues the follow up services.

The Consultancy arm is the key functional division which contributes to the financial sustainability of the organization. This division has two main arms; training and advisory service, and technological solutions. It provides technical advisory service on the key thematic areas and conducts training programmes on the same thematic areas. This is a demand-based service, and clients are required to pay for this service. Also it delivers technological services - basically the technological equipment and products. PASL has defined these products and services in its product and service booklet.

Sustainability is a multidimensional issue and financial sustainability is only one aspect of it. There are varieties of other factors threaten the sustainability of any knowledge service: developing and preserving digital collections, adopting new technologies, providing free services and lowering the ecological footprint (Jankowska, \& Marcum, (2010). While the consultancy arm focuses on achieving financial sustainability, the knowledge hub aims to address the other dimensions of sustainability as mentioned above. The Knowledge hub is the core of PASL's content. It comprises 3 main units; online knowledge services, publishing and bookshop, and knowledge avenue. Online knowledge services mainly consist of the web portal and the appropriate technology digital repository. It also consists of provision of knowledge services through social media such as Facebook, Twitter, You-Tube and mobile service such as provision of knowledge via Short Messaging Service (SMS). Practical Answers publishing develops and publishes knowledge material on appropriate technology based on its knowledge and experience. Practical Answers bookshop undertakes the sales of these publications. The Bookshop also undertakes the marketing and sales of publications of other publishers on commission basis. Knowledge Avenue is the place where currently the 
physical library of PASL is held. The plan is to expand this with the facility to play, share, collaborate and learn both formal and informally. The avenue will be built based on the green concept in which all environment aspects are taken in to consideration. These also contribute towards the financial sustainability of PASL.

Monitoring and Evaluation division is a functional division which develops monitoring and evaluation strategies and plans for PASL. It is very important to evaluate the effectiveness of internal practices and the impact created by PASL externally. The Marketing and promotion division develops and implements marketing and promotional strategies. Eventually it is intended that PASL will have its' own support unit to provide financial, administrative, and information technology infrastructure assistance for its operations. This is because at present PASL utilizes the services provided by Practical Actions financial, administrative and IT unit.

\subsection{Content, format and dissemination}

Practical Answers Sri Lanka holds a rich content base on appropriate technology gathered over 25 years and it is the key comparative advantage of PASL. Practical Answers Sri Lanka is continuously working on generating new content in various formats in different languages depending on the target audiences. While assuring the quality of the knowledge products, PASL disseminates these products through its web, digital repository, social media, mass media, bookshop and Knowledge Avenue.

\subsection{Marketing and sales strategy}

Practical Answers Sri Lanka's marketing strategy mainly focuses on creating awareness and communicates the value of PASL's offerings to the users who seeks the relevant knowledge, to the current and potential customers, to partners and to society in general. Marketing strategy defines PASL's products and value proposition to its clients and guides the positioning strategy aimed at strengthening the brand name and leveraging the competitive advantage. Identification of new business opportunities and client segments has been given an emphasis in the strategy (Daniels, Bhattacharya, Velliangiri, Zhang, Ali, 2013). Also it elaborates the promotion and advertising via direct mail, emails, and mass media and through exhibitions and fairs. Practical Answers Sri Lanka's 'product catalogues' carry a portfolio of its products and services, and PASL currently has a strong social media presence.

\subsection{Monitoring, evaluation \& impact assessment}

Adopting new strategies to assure the quality of the PASL service in the new business model identified as very important. For that purpose, PASL introduced new quality standards to assure the quality of the products and services and tracking systems to monitor its' operations in relation to its' goals and objectives, help the team better meet the targets.

When social impact assessments are undertaken with a spirit of inquiry, organizations can distinguish successful practices from unsuccessful ones by considering intended and unintended consequences and by factoring in external influences such as community support, beneficiary perceptions and donor expectations (London, 2009). Practical Answers Sri Lanka 
will adopt a new and in-depth social impact assessment framework to monitor and evaluate the impact that Practical Answers has on its beneficiaries by April 2014. Such a learning driven impact assessment is deemed especially important to Practical Action Sri Lanka as an aspiring social enterprise, in order to be mindful of setting both social goals as well as financial goals.

\subsection{Partnerships and networks}

Partnerships are particularly important to PASL to reach its mission and goals to create the expected social impact through knowledge sharing. PASL will partner with various government, nongovernmental and private sector partners to strengthen its content base (content partners), to disseminate the content (dissemination partners), to build strategic alliances (strategic partners) and to raise funds (financial partners/donors).

\subsection{Changing organizations culture}

Practical Answers Sri Lanka is making an extra effort to change the mindset of its' team and the culture of the organization as it is very important for successful transition to a social enterprise and to internalize the dual organizational goals (Daniels et al., 2013). Practical Answers Sri Lanka is also investing on building the capacity of its' team to acquire necessary skills and competencies (performance management, customer service, business communication, etc.) needed in order to function in a revenue-generating enterprise. Leading the PASL team in the right direction and rewarding them to motivate the team is also highly important.

\section{Key Lessons and Future Concerns}

\subsection{Business and Impact-oriented Culture:}

In order to continue serving the various client segments, providing them with technical information, and assisting them to grow their livelihoods, PASL has to adopt a more business-oriented culture (Daniels et al., 2013).. It drives PASL to achieve financial profitability, while continuing to maximize social impact.

\subsection{Leveraging relationships and building strategic alliances:}

The success of PASL towards achieving its' goals and objectives will heavily depend on how well it capitalizes the existing network of stakeholders, and how well it builds strategic alliances with potential partners.

\subsection{Attracting potential Donors and Investors:}

Along with the growth of PASL as a successful business model, strong networks of partners and social impact, it has to attract potential donors and impact investors to finance its' growth and future expansion (Daniels et al., 2013). 


\subsection{Creativity and innovation:}

In the current context of the rapidly increasing growth of knowledge and new technologies, it is vital that PASL must be constantly attuned to new opportunities to expand its' own knowledge and increase its services. This will require all members of PASL team to be constantly alert to such opportunities and build their own capacities to be creative and innovative.

\subsection{Changing role of the information workers:}

Earlier the key roles of the team members who were assign to handle information were to identify, select, format, organize and disseminate the content for the intended audiences. With the change of the business model the need to shoulder additional responsibilities as social entrepreneurs has become vital in addition to above mentioned traditional roles and responsibilities.

\section{Conclusion}

In conclusion, sustainability is a key challenge which any donor funded knowledge sharing service such as PASL has to face in the long term. Most institutions operating with donor funds tend to discontinue its' affiliated knowledge services or reduce finding allocations considering these as non-essential burdens to the institution. Hence, it is very important to continually investigate alternative strategies to ensure the sustainability of such knowledge services.

Facing the challenge of financial sustainability within a highly uncertain internal environment, PASL has been able to come up with a new hybrid business model in which PASL generates revenues by providing services in order to subsidize PASL's knowledge sharing service. Adopting new strategies, restructuring its' operations and services, introducing new services, developing a new marketing and promotional strategy are fundamental during the process of transformation.

The quality assurance of the knowledge materials and effective delivery services, creating new content formats and adopting new channels and technologies for dissemination of information and innovative concepts to present the information are highly important to maintain the competitiveness of the service in a challenging environment.

However PASL has to make an extra effort to change the mindset of the team and the culture to internalize the dual organizational goals. Also PASL has to build the capacity of the team to acquire the necessary skills and competencies. Further to that PASL has to implement the suggested impact monitoring framework in order to measure the impact of the service effectively. 


\section{References}

Daniels, D., Bhattacharya, P.,Velliangiri, A., Zhang, Y. \& Ali, H. (2013). Transforming nonprofit operations to revenue generating business models. Unpublished manuscript.

Jankowska, M.A., \&Marcum,J.W. (2010). Sustainability challenge for academic libraries: planning for the future research and collage libraries. College \& research libraries, 71( 2), 160-170. Retrieved from http://lmd.lk/archives/2011/March/brands.htm

London,T. (2009,May). Making better investments at the base of the pyramid: Creating value for all, Part II.Harvard Business Review, 87(5), 106-113.

Practical Action.(2012). Practical Answers group business plan. Unpublished manuscript.

Practical Action. (2012). Practical Answers Sri Lanka business plan. Unpublished manuscript 